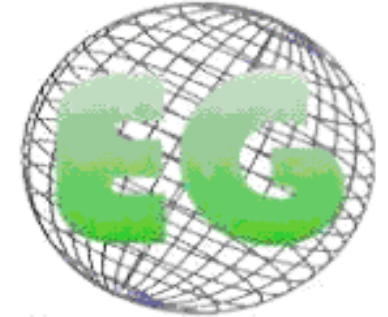

ISSN 1695-6141

$N^{\circ} 21$

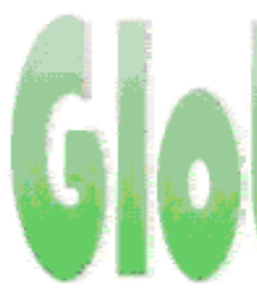

Revista electrónica trimestral de Enfermería

www.um.es/egloball

Enero 2011

DOCENCIA - INVESTIGACIÓN

\title{
ESTRATEGIA DE INTERVENCIÓN PARA EL CUIDADO DE LOS/AS ADOLESCENTES EN SALUD SEXUAL Y REPRODUCTIVA. POLICLÍNICO "DR. CARLOS J. FINLAY". MARIANAO, 2009.
}

STRATEGY OF INTERVENTION FOR THE CARE OF ADOLESCENTS IN SEXUAL AND REPRODUCTIVE HEALTH. CLINICIAN “DR. CARLOS J. FINLAY”. MARIANAO, 2009.

\section{${ }^{*}$ Fajardo Villarroel, Aurora}

*Licda. en Enfermería.Profesora Asistente. Institución: ICBP Victoria de Girón. La Habana. Cuba.

Palabras clave: Adolescencia, Sexualidad, Salud sexual y reproductiva, Cuidado.

Keywords: Adolescence, Sexuality, Sexual and reproductive health, Care.

\section{RESUMEN}

Se realizó un estudio descriptivo de corte transversal con el objetivo de diseñar una estrategia de intervención para el cuidado de los/as adolescentes en salud sexual y reproductiva que contribuya a elevar el conocimiento de estos, dirigido a los/as adolescentes del Consultorio \# 9 del Policlínico Docente Dr. Carlos J. Finlay en el periodo de enero-diciembre del 2009, municipio Marianao. La muestra quedó constituida por 43 adolescentes procedentes del consultorio, lo que constituyó el $100 \%$ del universo. Se aplicó un cuestionario sobre conocimiento en sexualidad. Dichos resultados fueron plasmados en tablas estadísticas y expresadas en frecuencias absolutas y relativas. Obteniendo como resultados la falta de instrumentación de programas sobre educación sexual en las áreas de salud, escuela e instituciones sociales, así como necesidades de aprendizajes como déficit de conocimientos en la utilización de los métodos anticonceptivos e insuficiente conocimiento sobre el aborto y sus posibles complicaciones médicas; también carecían de conocimiento sobre las ITS, y las repercusiones médicas y sociales del embarazo, la presencia de tabúes acerca de las orientaciones sexuales estuvo presente en esta investigación por lo que llegamos a la conclusión de que los programas de educación sexual aún son insuficientes en su puesta en marcha, tiene que producirse una revolución en la organización y aplicación de los mismos. La atención primaria de salud, escalón fundamental de esta labor educativa, tiene ante sí un nuevo terreno como un todo integrador en la educación sexual de las nuevas generaciones. 


\section{ABSTRACT}

A descriptive transversal study was carried out from January to December, 2009 aimed at designing an intervention strategy for teenagers' sexual and reproductive health that contributes to the knowledge of teenagers belonging to Doctor's Surgery number 9 of the Carlos J. Finlay clinic, in Marianao. The sample was 43 teenagers - $100 \%$ of the universe. A questionnaire on knowledge about sexuality was applied and the findings were recorded in statistical charts and expressed in absolute and relative frequencies. The findings revealed a lack of programs on sexual education in health areas, schools and social facilities, such as learning needs, as well as in knowledges about using contraceptive methods, abortion and it's complications, and knowledges about Sexually transmited diseases(STDs) pregnancy and taboos about sexual preferences. As a conclusion, sexual education programs are insufficient. Primary health attention is an important link in the sexual education of the new generations-

\section{INTRODUCCION}

Una década puede no significar más que unos pocos años incluidos en una larga vida. Sin embargo, una etapa tan breve como la adolescencia, termina por formar la personalidad de los seres humanos. Es en este período donde se experimentan cambios constantes y nuevas vivencias que pueden marcar a una persona de por vida. (1)

Hablar de la salud sexual y reproductiva en el/la adolescente lleva implícito una orientación hacia el desarrollo humano en su función reproductora, creativa e intelectual. La educación sexual forma parte de la educación general integral del individuo. La Msc. Mariela Castro Espín, en una entrevista al periódico "Juventud Rebelde" en el año 2000 expresó: ..."Este proceso es más largo que el de aprender a leer y escribir, pienso que todavía estamos en la etapa de alfabetización, nos encontramos en el ABC de la sexualidad"' (2-3).

A pesar de los múltiples esfuerzos desarrollados por nuestro gobierno y Sistema Nacional de Salud, todavía no son suficientes la puesta en marcha de los programas de educación sexual en nuestras áreas de salud, continúa la incidencia y prevalencia de infecciones de transmisión sexual, el incremento del embarazo en la adolescencia, la aparición de nuevos casos de seropositivos, sin hablar de los tabúes acerca de las orientaciones sexuales, son problemas que hemos podido constatar en diferentes áreas de salud. Nosotros abordaremos las relacionadas con el CM 9 perteneciente al Policlínico Docente Dr. Carlos J. Finlay del municipio Marianao.

El ser humano es el objeto central de la enfermería; aunque otras disciplinas sanitarias comparten esta preocupación, sus objetivos son distintos. El propósito de la especialidad es suministrar cuidados al individuo que carece de la fuerza, de la voluntad o de los conocimientos necesarios para atender a sus propias necesidades en materia de salud.

El trabajo de enfermería en cualquier nivel de atención constituye un eslabón fundamental para lograr el cuidado y la recuperación del paciente o la conservación de la salud de la persona supuestamente sana. Con el fin de alcanzar este objetivo es necesaria la puesta en marcha de estrategias de intervención para Promover cambios saludables a nivel grupal, institucional y comunitario donde la población sea la protagonista en la solución de los problemas de la vida cotidiana. (4).

El desarrollo de estrategias de intervención a través de talleres educativos con adolescentes es una herramienta eficaz para lograr un comportamiento sexual responsable en nuestros adolescentes y jóvenes, capaz de disfrutar de una sexualidad sana llena de placer y 
satisfacción. Para esta investigación nos proponemos como objetivo general diseñar una estrategia de intervención para el cuidado de los/as adolescentes en salud sexual y reproductiva que contribuya a elevar el conocimiento en cuanto a salud sexual y reproductiva, dirigido a los/as adolescentes del CM 9 del Policlínico Docente Dr. Carlos J. Finlay.

\section{MATERIAL Y MÉTODO}

Se realizó un estudio cuantitativo con un corte transversal de tipo descriptivo en el policlínico Dr. Carlos J Finlay del municipio Marianao, durante el año 2009. La muestra quedó constituida por 43 adolescentes procedentes del consultorio lo que supuso el $100 \%$ del universo. Se le aplicó un cuestionario sobre conocimiento en sexualidad. Dichos resultados fueron plasmados en tablas estadísticas y expresados en frecuencias absolutas y relativas.

En todos los casos se realizaron las respectivas pruebas de hipótesis, siendo el mínimo permisible de 0.05 .

\section{Operacionalización de las variables}

\section{$\underline{\text { Variable cuantitativa continúa }}$}

\section{Edad:}

Adolescencia intermedia: 15-17 años.

Adolescencia tardía: 18-19 años.

$\underline{\text { Variable cualitativa ordinales }}$

Sexo

Femenino_. 21

Masculino-.22

Niveles de conocimientos

Clave de calificación y escala de nivel según la puntuación que expresa el nivel de conocimientos que tienen los adolescentes sobre el tema.

\footnotetext{
$>$ Muy alto, de 15 a 13 respuestas satisfactorias.

$>$ Alto, de 12 a 10 respuestas satisfactorias.

$>$ Medio, de 9 a 7 respuestas satisfactorias.

$>$ Bajo, de 6 respuestas satisfactorias.
}

\section{ANÁLISIS DE LOS RESULTADOS}

En nuestro estudio, toda nuestra población es adolescente, a su totalidad se le practicó un cuestionario para, a través de éste, realizar el diagnóstico de las necesidades de aprendizaje 
en la esfera de la sexualidad y a partir de esas necesidades elaborar un programa de educación sexual.

Se les aplicó una encuesta a 43 adolescentes pertenecientes al CM 9 del Policlínico Docente DR. Carlos J. Finlay", de los cuales 21 corresponden al sexo femenino para un $48.8 \%$ y 22 al sexo masculino para un $51.2 \%$. Los adolescentes estaban comprendidos en las edades entre $15-19$ años.

Anexo I. Distribución de adolescentes encuestados por grupos de edades y sexo. CM 9 Policlínico Docentes Dr. Carlos J. Finlay.2009.

\begin{tabular}{|l|l|l|l|l|l|l|}
\hline Edad & $\begin{array}{l}\text { Sexo } \\
\text { Fem. }\end{array}$ & $\%$ & $\begin{array}{l}\text { Sexo } \\
\text { masc. }\end{array}$ & $\%$ & $\begin{array}{r}\text { To } \\
\text { tal }\end{array}$ & $\%$ \\
\hline $15-17$ & 11 & 26 & 10 & 23 & 21 & 49 \\
\hline $18-19$ & 10 & 23 & 12 & 28 & 22 & 51 \\
\hline Total & 21 & 49 & 22 & 51 & 43 & 100 \\
\hline
\end{tabular}

Fuente: Encuesta.

Sobre quién debía brindar educación sexual, 34 adolescentes respondió que debían ser los padres, para un $79.06 \%$, seis respondieron que la escuela, para un $13.95 \%$ y tres respondieron que a través de sus grupos de pares (amigos) para un 6.99\%.

Anexo 2. Información sobre educación sexual recibida.

CM 9 Policlínico Docentes Dr. Carlos J. Finlay. 2009.

\begin{tabular}{|l|l|l|}
\hline $\begin{array}{l}\text { Educación } \\
\text { Sexual } \\
\text { Recibida. }\end{array}$ & Total. & $\%$ \\
\hline Padres. & 34 & 79.06 \\
\hline Escuela. & 6 & 13.95 \\
\hline Amigos/as. & 3 & 6.99 \\
\hline Total & 43 & 100 \\
\hline
\end{tabular}

Fuente: Encuesta

La mayoría de los/as adolescentes plantearon que han recibido información sexual a través de los padres y pueden dirigirse a ellos en caso de inquietud de carácter sexual; sin embargo, el papel de la escuela ha resultado inferior, aspecto a tener en cuenta al diseñar programas de educación sexual; esto coincide con otros trabajos de investigación, relacionados con el tema de la adolescencia. 
Todos los/as adolescentes encuestados habían tenido ya su primera relación sexual y en esta solamente 8 para un $18.6 \%$ habían utilizado algún método anticonceptivo.

Anexo 3: Utilizacion de metodos anticonceptivos en la primera relacion sexual.

CM 9 Policlinico Docente Dr Carlos J Finlay.2009.

\begin{tabular}{|l|l|c|}
\hline $\begin{array}{l}\text { Primera } \\
\text { relacion } \\
\text { sexual. }\end{array}$ & Adolescentes. & $\%$ \\
\hline $\begin{array}{l}\text { Con metodo } \\
\text { anticonceptivo. }\end{array}$ & 8 & 18.6 \\
\hline $\begin{array}{l}\text { Sin metodo } \\
\text { anticonceptivo. }\end{array}$ & 35 & 81,4 \\
\hline Total & 43 & 100 \\
\hline
\end{tabular}

Fuente: Encuesta.

El método anticonceptivo más conocido por nuestros/as adolescentes es el condón, seguido de los dispositivos intra-uterinos (DIU) y tabletas anticonceptivas, pero existe una distancia entre el conocimiento y la utilización de los mismos y muy particular el condón, que a pesar de conocerlo, no todos lo utilizan, esto coincide con otras literaturas que tratan del tema. (5$6)$.

Acerca de la responsabilidad de la protección, 21 adolescentes para un $48.8 \%$ manifestaron que era de la mujer, 8 respondieron que del hombre para un $18.6 \%$ y solamente 14 para un $32.5 \%$ contestaron que de la pareja.

Anexo 4. Responsabilidad de la protección en la pareja.

CM 9 Policlinico Docente Dr Carlos J Finlay. 2009.

\begin{tabular}{|l|l|l|}
\hline Responsabilidad & No & \multicolumn{2}{|c|}{$\%$} \\
\hline Mujer & 21 & 48.8 \\
\hline Hombre & 8 & 18.6 \\
\hline Pareja & 14 & 32.5 \\
\hline Total & 43 & 100 \\
\hline
\end{tabular}

Fuente: Encuestados

Esto demuestra el pensamiento machista de los jóvenes heredado de generaciones pasadas. 
Sobre la utilización de algún método anticonceptivo, 37 adolescentes respondieron que no lo utilizaban, para un $86 \%$ y seis respondieron afirmativamente, para un $13.9 \%$, lo que demuestra que no son suficientes los conocimientos.

Alguno de los/as adolescentes encuestados no conocían las repercusiones sociales y médicas del embarazo en la adolescencia; esto lo tuvimos en cuenta a la hora de la elaboración del programa de educación sexual.

Anexo 5. Embarazo en la adolescencia. Repercusión social. CM 9 Policlinico Docente Dr Carlos J Finlay. 2009.

\begin{tabular}{|l|l|l|}
\hline Repercusión & No & $\%$ \\
\hline Conocian. & 13 & 30.3 \\
\hline $\begin{array}{l}\text { No } \\
\text { conocian. }\end{array}$ & 30 & 69.7 \\
\hline Total. & 43 & 100 \\
\hline
\end{tabular}

Fuente: Encuestados

Sobre si recibieron curso, talleres o seminarios del tema hasta el momento sobre educación sexual en la escuela, 10 de los encuestados manifestaron que sí, para un $23.3 \%$, los 33 restantes nunca han recibido orientación en materia de educación sexual en la escuela, para un $76.7 \%$.

Sobre la diversidad sexual, solamente 7 adolescentes plantearon que no es algo incorrecto, para un $16.2 \%$, el resto, 36 adolescentes expresaron que era incorrecto, para un $83.7 \%$, lo que demuestra el grado de desconocimiento sobre el tema.

A la luz de los resultados obtenidos han quedado demostradas las nececidades de aprendizajes de los/as adolescentes estudiados como son: deficit de conocimientos en la utilización de los métodos anticonceptivos, insuficiente conocimiento sobre el aborto y sus posibles complicaciones médicas, carecían de conocimiento sobre las infecciones de transmisión sexual, y las repercusiones médicas - sociales del embarazo en la adolescencia, como la presencia de tabúes acerca de las orientaciones sexuales.

Sistematizar los indicadores diagnóstico que se viven en la actualidad en este grupo poblacional sería un sueño para el futuro saludable de nuestros adolescentes para posteriormente los expertos en promoción de salud diseñen programas de estrategias de intervención comunitaria donde estos logren ser sujetos verdaderamente sanos en la medida que se apropien de su realidad cotidiana y que sean capaces de transformarla y transformarse (7-8).

\section{DISCUSIÓN}

Si bien es cierto que nuestro sistema nacional de salud une esfuerzos en lograr una salud sexual y reproductiva de las nuevas generaciones, es una realidad social y profesional que nos toca a los especialista de las ciencias de la enfermería educar a padres, familias, maestros e instituciones sobre este complejo tema. 
Como paso hacia el futuro, el cuidado no es posible en la práctica sin una transformación de nuestro imaginario social hacia una apertura de nuevas concepciones en el crecimiento de nuestra subjetividad.

Investigaciones realizadas en New York confirman que el $57 \%$ de los adolescentes estudiados sufrian de una insatisfacción cognitiva en conocimientos de sexualidad, confirman una gran parte de los resultados que la prevalencia en déficit de conocimientos del tema en jóvenes masculinos era superior que en las jóvenes, pero ambos sexos carecían de la utilización de métodos anticonceptivos en la primera relación sexual, por lo que se plantea que de ocho de cada diez chicas se infectaban con el virus del papiloma humano en la primera relación sexual en EE:UU .(9-10).

También en dicha investigación coincide que los adolescentes reciben mayor información del tema por la familia, no así por la escuela variable que es igual a la nuestra; observamos que los adolescentes cubanos presentan más tabues en relación a las orientaciones sexoeróticas debido a nuestra formación machista, no así en paises europeos (11-12) Los hallazgos del presente trabajo realizado y las búsquedas realizadas por otros autores sugieren que se investigue y se trabaje sobre el tema por lo que podemos finalizar expresando que la educación sexual es responsabilidad de toda la sociedad, representa un complejo proceso social que debe ser consciente y sistemático, le corresponde fundamentalmente por su labor educativa a los profesionales de la enfermería transmitir a las nuevas generaciones conocimientos claros, valorando que toda persona por joven que sea, tiene derecho a una adecuada y feliz educación sexual.

Los programas de educación sexual aún son insuficientes mundialmente, tiene que producirse una revolución en la organización de su aplicación. A la atención primaria de salud le corresponde fundamentalmente esta labor educativa, y tiene ante sí un nuevo terreno que recorrer como un todo integrador en la educación sexual de las nuevas generaciones.

\section{CONCLUSIONES}

La educación sexual es responsabilidad de toda la sociedad, es un complejo proceso social, demostrándose ser una herramienta eficaz en las transformaciones de estilos de vida de los/as adolescentes. La inclusión de programas educativos en salud sexual y reproductiva debe ser una tarea continua, sistemática, en función de las necesidades del educando y ofrecerles a estos un papel activo y protagónico en la apropiación del conocimiento.

Teniendo en cuenta lo planteado en nuestro trabajo exponemos a continuación el programa de intervención comunitaria, el cual es puesto en práctica por nuestros profesionales de enfermería en nuestra área de salud en la actualidad. Su evaluación e impacto serán tema de otro proyecto de investigación y exhortamos a la comunidad científica de enfermería a trabajar en este importante tema.

\section{Programa de intervención comunitaria para el cuidado de los/as adolescentes en salud sexual y reproductiva}

\section{Fundamentación teórica:}

Teniendo en cuenta que la educación sexual es un proceso que ocurre a lo largo de la vida, es evidente que les corresponde a los profesionales de enfermería en la Atención primaria de salud aportar desde nuestra responsabilidad del cuidado la educación de la sexualidad 
de las nuevas generaciones como categoría del crecimiento humano. De este modo y como profesionales autónomos, pero con un importante rol multidisciplinario debemos exponer nuestros instrumentos desde intervención comunitaria para reconocer esta problemática educativas y aportar elementos enriquecedores a los/as adolescentes en su crecimiento sexual y humano.

Objetivo general: Capacitar en salud sexual y reproductiva la etapa de la adolescencia en un área de salud.

Organización: El programa de intervención comunitaria para el cuidado de los/as adolescentes en salud sexual y reproductiva utilizará técnicas basadas en la participación activa de los/as involucrados/as en este proceso. Se utilizan materiales didácticos como: folletos, computadoras, películas, etc.

El taller se encuentra planificado para ser impartido en dos frecuencias por semana, con un tiempo de duración total de 30 horas. Se utilizando métodos preventivos de enseñanza a predominio grupal. Los contenidos educativos a tratar deben ir acorde al desarrollo psicosexual de los adolescentes. Se aconseja dividir los grupos según las edades.

- $15-17$ años

- 18 - 19 años

El programa incluye los siguientes temas:

\section{Plan temático.}

Tema I: La adolescencia, una etapa del ciclo vital de la vida.

- Características generales del desarrollo.

- Anatomía y Fisiología sexual.

- Aparato reproductor femenino.

- Aparato reproductor masculino.

- Ciclo de la respuesta sexual.

\section{Tema II: Diversidad}

- Orientaciones sexuales. Tabúes vs respeto de todos/as.

- Homosexualidad.

- Bisexualidad.

- Heterosexualidad.

- Transexualismo.

Tema III: Problema Actual de salud. Infecciones de transmisión sexual.

- Infecciones de transmisión sexual.

- VIH / SIDA. Pandemia Mundial.

- El sexo seguro.

\section{Tema IV: Salud reproductiva}

- Embarazo en la adolescencia. 
- Consecuencias médico - social del embarazo en la adolescencia.

- Paternidad y maternidad concientes.

- Parto y Puerperio.

- Métodos anticonceptivos. Como emplearlos correctamente.

- Planificación familiar.

\section{Tema V: Nuestras relaciones con todos/as}

- Familia.

- La escuela.

- El grupo.

- Las relaciones de pareja.

- Grupos de Apoyo.

Evaluación del programa: Se realizará de forma sistemática y parcial estimulando el trabajo en grupo con los/as adolescentes, concluimos con alguna iniciativa de los/as participantes donde apliquen lo aprendido.

\section{REFERENCIAS BIBLIOGRÁFICAS}

1. Albert Sobrio S, Albert Sobrio M, Friebel Paradela E. imagen corporal y diagnóstico en Enfermería en adolescentes. Rev metas de enferm mar 2005; 8(2): 50-57.

2. Adolescencia. Salud reproductiva. Boletín Bimestral de la Unidad de Investigación Epidemiológica y en Servicios de Salud del Adolescente con el Patrimonio de la OPS. Año 2, No. 3. Guadalajara. Mayo-Junio 5. 2000.

3. Periódico Juventud Rebelde. La Habana, 5 de Abril del 2000.Pp 6.

4. Díaz Bravo, C. (2007). "La educación de la sexualidad en un mundo mediático. Reflexiones desde Cuba». Sexología y Sociedad, La Habana, año 13, no. 35, pp. 26-30.

5.Rigol Ricardo, O y Colectivos de Autores. Obstetricia y Ginecología, editorial ciencias médicas, 2004.

6. PELÁEZ M., J. Anticoncepción de emergencia en adolescentes: nuestra experiencia. Revista de sexología y sociedad. Año 14, No. 37, Agosto de 2008.

7. González, A. y b. Castellanos (2003). Sexualidad y géneros. Alternativas para su educación ante los retos del siglo XXI. Ed. Científico-Técnica, La Habana.

8. Guerrero, P. (1998). «Pasado, presente y futuro de la educación sexual en Colombia». Revista de Psiquiatría, vol. XXVII, no.4.

9. Boonstra, H. (2004). Abstinence Promotion and the U.S. Approach to HIV/AIDS Prevention Overseas. The Alan Guttmacher Institute, New York.

10. Kempnec, M. A. (2004). The Politics of Sexuality Education. Sexuality Information and Education Council of the US (SIECUS), Washington. 
11. Bianco, Mabel y F. Aranda (2007). Las mujeres no esperamos más. Acabemos la violencia contra la mujer y el VIH/SIDA ya. Publicación para América Latina y el Caribe. Buenos Aires. Fondo de Desarrollo de las Naciones Unidas para la Mujer (2006). Información sobre el VIH y el SIDA. Especial para mujeres. UNIFEM-México.

12. Berlinguer, G. (2007). «Determinantes sociales de las enfermedades». Rev. Cubana Salud Pública, vol. 33, no. 1. 


\section{CUESTIONARIO}

Te pedimos que leas detenidamente cada pregunta y que tus respuestas sean lo más sinceras posibles.
( helado preferido)
Gracias.
(color preferido)

1. Edad:

Sexo:

Grado:

2. ¿Quién debería brindar información sobre educación sexual?

Madre o padre miembros de la familia

Escuela Amigos

Médico y enfermera de familia

Medios de difusión masiva

Todos los anteriores.

Trabajador social

3. ¿Es suficiente la educación sexual que se imparte en la escuela actualmente? Si No insuficiente

4. ¿Te brindan información sobre educación sexual tus padres?

Si_ No

5. A quienes te diriges con plena confianza cada vez que tienes una inquietud de carácter sexual.

Padres__Escuela_ Médico y enfermera de familia_ Amistades

Todos los anteriores

6. En la primera relación sexual con penetración tú o tu pareja utilizaron algún método anticonceptivo. Si No La responsabilidad es

7. Menciona los métodos anticonceptivos que conoces para evitar un embarazo.

8. ¿Es el aborto un método anticonceptivo?
Si ¿Por qué?
No ¿Por qué?

9. Cuales son las posibles complicaciones que pueden presentarse durante o posterior a la interrupción del embarazo.

¿Cuáles son?

10. ¿Conoce Ud. las repercusiones sociales y médicas del embarazo?

$\mathrm{Si}$

No

¿Cuáles son?

11. Conoce Ud las infecciones de transmisión sexual.

$\mathrm{Si}$

¿Cuáles?

No 
12. ¿Has recibido en tu escuela algún curso, taller, seminario de educación sexual? $\mathrm{Si}$ No

13. ¿Consideras la diversidad sexual algo incorrecto? $\mathrm{Si}$ No ¿por qué?

Clave de calificación y escala de nivel según la puntuación que expresa el nivel de conocimientos que tienen los adolescentes sobre el tema.

Muy alto de 15 a 13 respuestas satisfactorias.

> Alto de 12 a 10 respuestas satisfactorias.

$>$ Medio de 9 a 7 respuestas satisfactorias.

$>$ Bajo de 6 respuestas satisfactorias. 